\title{
Thyrotoxicosis following SARS-COV-2 vaccination: a case series and discussion
}

\author{
B. Pla Peris ${ }^{1}$ - A. Á. Merchante Alfaro ${ }^{1,2} \cdot$ F. J. Maravall Royo ${ }^{1,2} \cdot$ P. Abellán Galiana ${ }^{1,3} \cdot$ S. Pérez Naranjo ${ }^{1}$. \\ M. González Boillos ${ }^{1}$
}

Received: 8 December 2021 / Accepted: 30 December 2021 / Published online: 11 January 2022

(c) Italian Society of Endocrinology (SIE) 2022

\begin{abstract}
Aim To describe a case series of thyrotoxicosis likely triggered by SARS-CoV-2 vaccination and to warn physicians about this potential correlation. To report clinical, laboratory and imaging findings and provide further information that goes in line with the underlying mechanisms.

Methods Single-center case series based on all the information collected in the hospital medical records, as well as the temporal sequence between the onset of symptoms and COVID-19 vaccination.

Results We report 8 cases with thyrotoxicosis after SARS-CoV-2 vaccination. 4 cases of Graves' disease (GD), 2 cases of subacute painful thyroiditis (SAT), 1 case of concurrent GD and SAT and 1 case of atypical subacute thyroiditis. Five patients received BNT162b2 mRNA vaccine, 3 patients 1273 mRNA vaccine. The onset of symptoms following vaccination ranged from 10 to 14 days in six of eight patients and from 7 to 8 weeks in two patients.

Conclusions Several hypotheses have been proposed to explain the potential correlation between SARS-CoV-2 vaccination and thyrotoxicosis, including immune system hyper-stimulation, molecular mimicry and Autoimmune/Autoinflammatory Syndrome Induced by Adjuvants (ASIA). We should pay greater attention to thyroid disorders in patients receiving vaccine against SARS-CoV-2.
\end{abstract}

Keywords SARS-CoV-2 vaccination · Thyrotoxicosis · Subacute painful thyroiditis · Graves' disease $\cdot$ Autoimmune syndrome $\cdot$ Adjuvants

\section{Introduction}

COVID-19 pandemic is one of the greatest challenges that the world faces. Since its emergence as a global pandemic, global COVID-19 death toll surpasses 5 million [1]. As the pandemic has progressed, it has also become increasingly well known that SARS-COV-2 affects not only the respiratory system, but also a wide spectrum of organs, including the endocrine system. In this regard, thyroid disorders,

\section{B. Pla Peris}

bplaperis@gmail.com

1 Department of Endocrinology and Nutrition, Hospital

General Universitario de Castellón, Avinguda de

Benicàssim, 128, 12004 Castelló de la Plana, Castelló, Spain

2 Departament of Medicine, Jaume I University, Castelló de la Plana, Castelló, Spain

3 Departament of Medicine, Cardenal Herrera-CEU University, Castelló de la Plana, Castelló, Spain phospho-calcium metabolism disorders and even severe diabetic ketoacidosis in individuals with new-onset diabetes have been reported [2-4].

In addition, and despite their lower prevalence, potential cases of thyroid disorders following SARS-CoV-2 vaccination are becoming more frequent and have been reported recently $[5,6]$.

The present study describes a case series of thyrotoxicosis likely triggered by SARS-CoV-2 vaccination in the period between May and September 2021. Our case series provide further information that goes in line with the underlying mechanisms proposed to explain SARS-CoV-2 vaccination and thyroid disorders correlation. 


\section{Materials and methods}

A single-center case series of eight adult patients who presented with thyrotoxicosis after SARS CoV-2 vaccination in the period between May and September 2021.

We aim to report demographic, clinical, laboratory and imaging findings, using data collected in the hospital medical records, as well as the temporal sequence between the onset of symptoms and COVID-19 vaccination.

\section{Results}

\section{Baseline characteristics}

Eight cases with thyrotoxicosis after SARS-CoV-2 vaccination in the period between May 2021 and September 2021. Five were females, the mean age was 56.6 years, ranging from 42 to 71 years. Seven of eight patients had normal thyroid function before vaccination, with no data of thyroid antibodies. None of the eight cases had family history of thyroid disease. One patient had subclinical hypothyroidism, with no data of thyroid antibodies. Five patients had received Pfizer BNT162b2 mRNA vaccine while the other three patients had received Moderna 1273 mRNA vaccine (Table 1).

\section{Biochemical diagnosis and clinical presentation}

We identified eight patients with thyrotoxicosis after SARS$\mathrm{CoV}-2$ vaccination. None of these patients reported any previous history of viral upper respiratory infection. Mean free $\mathrm{T} 4$ value was $3.28 \pm 1.12 \mathrm{ng} / \mathrm{dl}$ [0.54-1.24] with suppressed TSH. two patients (cases 4 and 6) were referred for evaluation of neck pain radiating to ears, asthenia, mild fever and clinical picture suggestive of thyrotoxicosis with raised C-reactive protein (CRP) and erythrocyte sedimentation rate (ESR), consistent with subacute painful thyroiditis (SAT). In five patients (1, 5, 7 and 8) TSH-receptor antibodies were positive (mean TSHR-Ab $4.05 \pm 0.83 \mathrm{U} / \mathrm{L}[N<1.75]$ ), acute phase reactants (ESR/CRP) were negative and symptoms were suggestive of Graves' disease (GD). One patient (case 3) underwent symptoms consistent with atypical subacute thyroiditis with no pain or swelling but clinical picture suggestive of thyrotoxicosis, mild fever, raise CRP $(30 \mathrm{mg} / \mathrm{dl}$ $[N<10)$ and ESR $(88 \mathrm{~mm} / \mathrm{h}[N<10])$ and negative TSHR$\mathrm{Ab}$. In one patient (case 2) Graves's disease and subacute painful thyroiditis coexisted and were concurrent (TSHRAb $3.8 \mathrm{U} / \mathrm{L}$, CRP $120 \mathrm{mg} / \mathrm{dl}$ and ESR $75 \mathrm{~mm} / \mathrm{h}$ ), with neck pain and mild fever, and clinical symptoms suggestive of thyrotoxicosis, including weight loss, palpitation and hand tremor. In this patient urine and respiratory infections were excluded. None of the patients with Graves' disease diagnosis had Graves' orbitopathy. No significant differences by age, gender or vaccine were observed. All laboratory results and clinical features of the eight cases are shown in Table 1 .

\section{Symptom's onset}

Symptoms developed after the first dose in six of the eight cases (cases 2, 3, 4, 5, 6 and 8). In the two patients that symptoms developed after the second dose (cases 1 and 7), the diagnosis was Graves's disease. Concerning the onset of symptoms following vaccination, it ranged from 10 to 14 days in six of eight patients, and from 7 to 8 weeks in two patients (Table 1). These two patients' diagnoses were Graves' disease, and both were vaccinated with Pfizer mRNA-BNT162b2 vaccine.

\section{Imaging studies}

Thyroid ultrasound was available at the time of thyrotoxicosis in seven patients. In the two cases of subacute painful thyroiditis (cases 4 and 6) thyroid ultrasound revealed an unstructured thyroid with diffuse hypoechoic areas and with diffuse hypovascularization. The three patients with Graves' disease and an available thyroid ultrasound at the time of thyrotoxicosis (cases 1, 5 and 7) had an enlarged thyroid with increased vascularity. In patient 3 (atypical thyroiditis diagnosis) thyroid ultrasound revealed heterogeneous echogenicity with diffuse hypoechoic areas and hypovascularization. In patient 2 (concurrent GD and SAT), thyroid ultrasound was only available 5 weeks after the onset of symptoms, thyroid was enlarged with heterogeneous echogenicity and diffuse hypoechoic pattern. Thyroid iodine scan revealed a diffuse markedly increased uptake over both lobes in patients with GD diagnosis (cases 1 and 5), and a decrease uptake in patients with SAT (cases 4 and 6), not being available at the time of thyrotoxicosis in all other cases. Imaging results are shown in Table 1.

\section{Treatment and patient follow up}

SAT patients (cases 4 and 6) and patient with atypical subacute thyroiditis diagnosis (case 3 ) were treated with nonsteroidal anti-inflammatory drugs. Twoweeks later, cases 4 and 6 medical examination revealed an improvement in hyperthyroidism with a decrease in acute phase reactants, and 4 and 5 weeks after clinical onset (respectively), subclinical hypothyroidism was established in case 4 and thyroid function was normal in case 6 . Patient 3 follow-up at week 6 revealed a subclinical hypothyroidism and normalization of APR, with no symptoms and no indication of treatment at this stage. Patients with diagnosis of Graves' disease (cases 


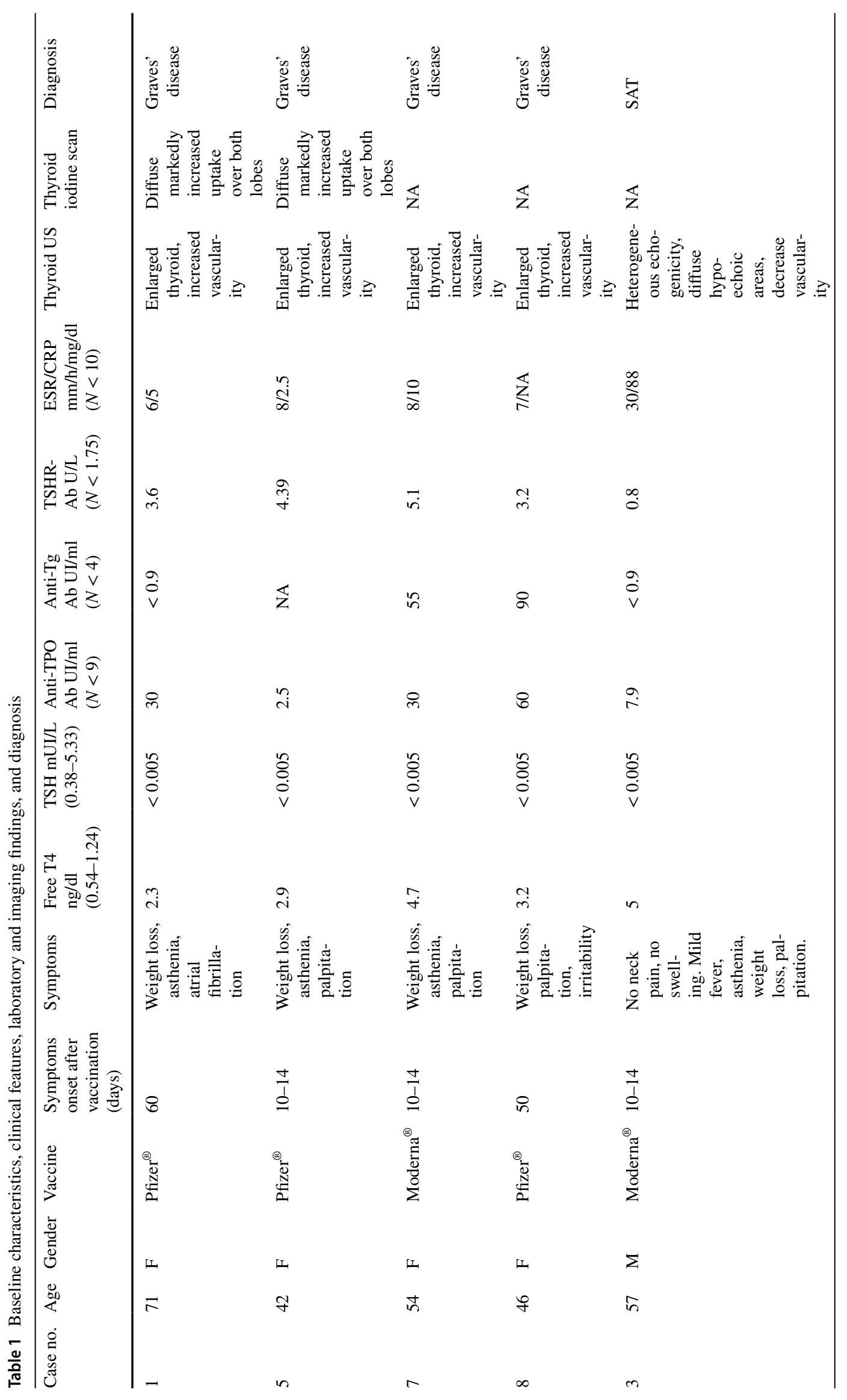




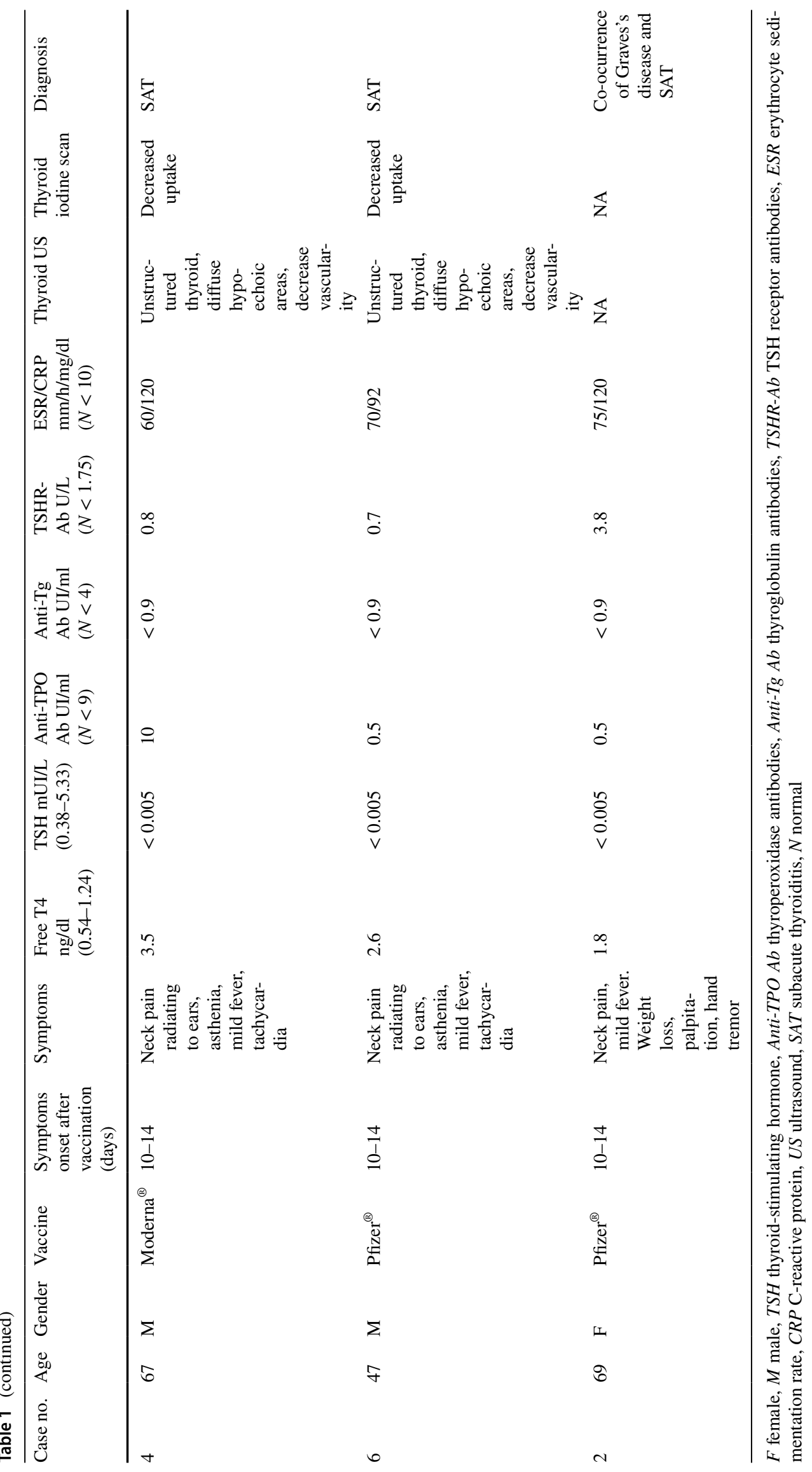


1, 5, 7 and 8) and patient with concurrent Graves's disease and subacute painful thyroiditis diagnosis (case 2) underwent treatment with antithyroid drugs (methimazole), and methimazole and non-steroidal anti-inflammatory drugs, respectively; with progressive dose reduction, in line with thyroid function improvement and symptoms. In cases 1 and 5 the presence of TSHR-Ab remained positive 2 months later; nevertheless, serum levels were lower than previous values (from 3.6 to $1.9 \mathrm{U} / \mathrm{L}$ and from 4.39 to $2.1 \mathrm{U} / \mathrm{L}$, respectively). TSHR-Ab evolution data in cases 2,7 and 8 are not available yet. All patients are still being followed up.

\section{Discussion}

Several hypotheses have been proposed to explain how SARS-CoV-2 may trigger thyroid disorders including the systemic immune-inflammatory response, and next to SARS-CoV-2 infection, SARS-CoV-2 vaccination seems to share a common mechanism.

An increasing number of series, both including overt subacute thyroiditis and atypical thyroiditis $[7,8]$ as well as thyroid autoimmune disease after COVID-19 infection $[9,10]$ has been described in the past year. It is noteworthy that SAT has been highlighted as a plausible underestimated manifestation of COVID-19 [2].

The mechanism by which COVID-19 induces thyroid dysfunction involves different mechanisms that can produce damage and alter endocrine function, including a direct action of the virus in thyroid tissue and an immunological response against the virus.

First and well-known, SARS-CoV-2 Spike Protein (S protein) binds ACE2 receptor, which has an important expression in several endocrine tissues including thyroid, leading to an increase of thyroid disorders in subjects with COVID19 infection [11]. Therefore, thyrotoxicosis could result from SARS-CoV-2 direct action on thyroid gland, as described in other viral infections.

It is noteworthy that, as recently described, SARS-CoV-2 infection can lead to both overt subacute thyroiditis consisting of typical presentation with neck pain radiating to ears, myalgia, mild fever and thyrotoxicosis with raised C-reactive protein (CRP)/erythrocyte sedimentation rate (ESR); but also, to a non-classical/atypical subacute thyroiditis with no pain or swelling but thyrotoxicosis and an increase of acute-phase reactants [12] described in up to one third of patients [8].

Alternatively, thyrotoxicosis could also result from an increased thyroid autoimmunity after SARS-CoV-2, and Graves' disease could be due to an immunological cascaded triggered by the acute inflammatory response to SARS$\mathrm{CoV}-2$, including the cytokine storm.
The inflammatory phenomenon during the infection has been described to be mediated by a cytokine release syndrome that takes place and leads to a significant increase in circulating IL-6, IL1RA, CCL2 and CCL8 (which recruit monocytes and macrophages) and CXCL9 and CXCL16 [chemoattractants of $\mathrm{T}$ or natural killer (NK) cells] [13]. It is noteworthy that an increase level of IL6 have already been reported in Graves' disease [14].

As with SARS-CoV-2 infection, different forms of thyrotoxicosis after SARS-CoV-2 vaccination have been described, including overt subacute thyroiditis and atypical thyroiditis, as well as autoimmune hyperthyroidism (Graves' disease) [5, 15], as we show in our case series. The general features of these are similar to thyrotoxicosis cases reported in SARS-CoV-2 infection. Most of these forms of thyrotoxicosis have been reported with mRNA-based vaccines, what goes in line with our case-series, although we have to point out that some cases have also been described after inactivated SARS-CoV2 and vector-based vaccines $[16,17]$. Nonetheless, it is important to highlight that in some patients, symptoms related with thyroiditis may have been identified as post-vaccination symptoms and, in consequence, many thyroiditis diagnoses may have been overlooked. Further investigations are needed to clarify these important concerns such as which is the real risk of thyroiditis depending on COVID-19 vaccine types.

Concerning Graves' disease, in our case series none of the patients had Graves' orbitopathy, albeit it has been described in one patient in the literature, with the hypothesis that COVID-19 could have triggered an autoimmune response against thyroid and ocular antigens, through molecular mechanisms underlying the action of the virus itself, as well as through the induction of a long-lasting stress status [10].

Two mechanisms for the association between SARSCoV-2 vaccination and thyroid disorders have been proposed.

First, immune-mediated complications after SARSCoV-2 infection are induced by immune system hyper-stimulation and molecular mimicry between the human proteome and SARS-CoV-2 components [15]. S protein has become a major target for the development of SARS-CoV2 vaccine. The majority of vaccines against SARS-CoV-2 encode the viral spike protein, employing it as the antigen to lead to a protective immune response against SARS-CoV-2. Recent studies $[16,18]$ suggest that thyroid peroxidase peptide sequences in thyroid tissue share similarity with SARSCoV-2 proteins. This could contribute to a cross-recognition between the modified SARS-CoV-2 spike protein encoded in RNA vaccine and the thyroid target proteins, due to molecular mimicry, leading to an autoimmune thyroiditis.

Second, the exposure to vaccine adjuvants used as immunogenicity enhancing agents of the vaccine formulation [19, $20]$ has been proposed to be capable of inducing diverse 
immune reactions $[16,21,22]$. This leads to the so-called Autoimmune/Autoinflammatory Syndrome Induced by Adjuvants (ASIA). It is difficult to ascertain the accuracy of this hypothetical autoimmune disorder as a pathogenic link between vaccination and thyrotoxicosis and it cannot be considered as a universally accepted pathogenic mechanism. Nevertheless, and on the basis of the literature review, it has been one of the most frequently repeated postulations. In this context, it is worth mentioning that at low rates and despite that case-control studies are still scarce, HPV vaccine has been related to primary ovarian failure [23] and to Guillain-Barré Syndrome [24], albeit not implying a causal association. The difficulty in proving a causal relationship between the clinical manifestations of the diseases and the use of adjuvants in vaccines should be noted since several studies do not confirm the role of adjuvants in the development of autoimmune diseases [25].

With regard to time interval after vaccination, as recently reported in other author case series [5], subacute thyroiditis after SARS-CoV-2 vaccination took place 10-14 days after vaccination. The described time course goes in line with the majority of patients in our case series, with an onset of symptoms 10-14 days after vaccination. In two patients of our case series the onset of symptoms ranged from 50 to 60 days. These two patients' diagnoses were Graves's disease. Literature review concerning cases reports in different countries regarding thyroid dysfunction after SARS-CoV-2 vaccination has shown a maximum time of onset after vaccination of 56 days [21]. In contrast and as it has recently been described [10], subacute thyroiditis triggered not by COVID-19 vaccination but by COVID-19 infection, seemed to take place 2-3 weeks after viral infection, and classic subacute thyroiditis (postviral) usually occurs within 2-8 weeks following viral infection [16].

Last, we have to emphasize that considering the high number of vaccine doses administered at the same period of time (May-September 2021), eight cases of thyrotoxicosis in 5 months is a relatively limited number. By October 2021 and following the Spanish vaccination strategy, $87.9 \%$ of the population over 12 years of age was already fully vaccinated [26]. In this context, we looked for the diagnoses of thyrotoxicosis performed in our Centre in the same period, resulting in 56. All 56 cases with a confirmed diagnosis of thyrotoxicosis had received at least one dose of the vaccine. Nevertheless, in no case did thyrotoxicosis occur before 8 weeks after vaccine administration, in contrast to our case series and to other cases in the literature. In any case, we would like to point out that despite the temporal sequence between the administration of the vaccine and the onset of symptoms, as well as the increasing number of cases being reported, the association in at least some of the described cases might be casual and simply chronologically associated with, albeit not caused by vaccination. In addition to this, it is noteworthy that following World Health Organization Immunization Safety Surveillance guidelines, an adverse event following immunization (AEFI) is defined as any untoward medical occurrence which follows immunization, which does not necessarily have a causal relationship with the usage of the vaccine [27]. Therefore, it is important to point out that thyroid disorders following COVID-19 vaccination represent a clinical event with a reasonable time relationship to vaccine administration, but that does not imply a cause-effect relationship [25].

Further research is needed to investigate the prevalence and pathogenesis of thyroid dysfunction following vaccination against COVID-19. Nonetheless and as a final thought, we truly believe that we must be aware that the SARS-CoV-2 vaccination may precipitate a thyrotoxicosis with different underlying etiologies. This may include overt subacute thyroiditis, atypical thyroiditis, and autoimmune hyperthyroidism, like in our case series, with concurrence of GD and SAT being possible. Therefore, based on our case series and as previously reported in the literature, we should pay greater attention to potential thyroid disorders in patients receiving vaccine against SARS-CoV-2 and closely monitor thyroid function in these patients.

\section{Declarations}

Conflict of interest None.

Ethical approval No animal studies are presented in this manuscript. No human studies are presented in this manuscript.

Informed consent Written informed consent was obtained from the individual(s) for the publication of any data included in this article.

\section{References}

1. https://www.who.int/emergencies/diseases/novel-coronavirus2019/situation-reports. Coronavirus disease (COVID-19) Weekly Epidemiological Update and Weekly Operational Update. Last day access 04/11/21

2. Brancatella A, Ricci D, Cappellani D et al (2020) Is subacute thyroiditis an underestimated manifestation of SARS-CoV-2 infection? Insights from a case series. J Clin Endocrinol Metab. https:// doi.org/10.1210/clinem/dgaa537

3. Bossoni S, Chiesa L, Giustina A (2020) Severe hypocalcemia in a thyroidectomized woman with COVID-19 infection. Endocrine 68(2):253-254. https://doi.org/10.1007/s12020-020-02326-0

4. Boddu SK, Aurangabadkar G, Kuchay MS (2020) New onset diabetes, type 1 diabetes and COVID-19. Diabetes Metab Syndr 14(6):2211-2217. https://doi.org/10.1016/j.dsx.2020.11.012

5. Lee KA, Kim YJ, Jin HY (2021) Thyrotoxicosis after COVID-19 vaccination: seven case reports and a literature review. Endocrine. https://doi.org/10.1007/s12020-021-02898-5

6. Hannah Ritchie, Edouard Mathieu, Lucas Rodés-Guirao, Cameron Appel, Charlie Giattino, Esteban Ortiz-Ospina, Joe Hasell, Bobbie Macdonald, Diana Beltekian and Max Roser 
(2020)_ “Coronavirus Pandemic (COVID-19)". Published online at OurWorldInData.org. Retrieved from: https://ourworldindata. org/coronavirus [Online Resource]. Last day access 04/11/21

7. Brancatella A, Ricci D, Viola N, Sgrò D, Santini F, Latrofa F (2020) Subacute thyroiditis after Sars-COV-2 infection. J Clin Endocrinol Metab. https://doi.org/10.1210/clinem/dgaa276

8. Garg MK, Gopalakrishnan M, Yadav P, Misra S (2020) Endocrine involvement in COVID-19: mechanisms, clinical features, and implications for care. Indian J Endocrinol Metab 24(5):381-386. https://doi.org/10.4103/ijem.IJEM_440_20

9. Jiménez-Blanco S, Pla-Peris B, Marazuela M (2021) COVID-19: a cause of recurrent Graves' hyperthyroidism? J Endocrinol Invest 44(2):387-388. https://doi.org/10.1007/s40618-020-01440-0

10. Lanzolla G, Marcocci C, Marinò M (2021) Graves' disease and Graves' orbitopathy following COVID-19. J Endocrinol Invest 44(9):2011-2012. https://doi.org/10.1007/s40618-021-01576-7

11. Marazuela M, Giustina A, Puig-Domingo M (2020) Endocrine and metabolic aspects of the COVID-19 pandemic [published correction appears in Rev Endocr Metab Disord. 2021 Mar; 22(1):145]. Rev Endocr Metab Disord 21(4):495-507. https:// doi.org/10.1007/s11154-020-09569-2

12. Muller I, Cannavaro D, Dazzi D et al (2020) SARS-CoV-2-related atypical thyroiditis. Lancet Diabetes Endocrinol 8(9):739-741. https://doi.org/10.1016/S2213-8587(20)30266-7

13. Blanco-Melo D, Nilsson-Payant BE, Liu WC et al (2020) Imbalanced host response to SARS-CoV-2 drives development of COVID-19. Cell 181(5):1036-1045.e9. https://doi.org/10.1016/j. cell.2020.04.026

14. Montebello A (2021) Recurrent Graves' disease post SARSCoV-2 infection. BMJ Case Rep 14(8):e244714. https://doi.org/ 10.1136/bcr-2021-244714

15. Siolos A, Gartzonika K, Tigas S (2021) Thyroiditis following vaccination against COVID-19: report of two cases and review of the literature. Metabol Open 12:100136. https://doi.org/10.1016/j. metop.2021.100136

16. İremli BG, Şendur SN, Ünlütürk U (2021) Three cases of subacute thyroiditis following SARS-CoV-2 vaccine: post-vaccination ASIA syndrome. J Clin Endocrinol Metab. https://doi.org/10. 1210/clinem/dgab373

17. Bornemann C, Woyk K, Bouter C (2021) Case report: two cases of subacute thyroiditis following SARS-CoV-2 vaccination. Front Med (Lausanne). 8:737142. https://doi.org/10.3389/fmed.2021. 737142
18. Oyibo SO (2021) Subacute thyroiditis after receiving the adenovirus-vectored vaccine for coronavirus disease (COVID-19). Cureus 13(6):e16045. https://doi.org/10.7759/cureus.16045

19. Watad A, David P, Brown S, Shoenfeld Y (2017) Autoimmune/ inflammatory syndrome induced by adjuvants and thyroid autoimmunity. Front Endocrinol (Lausanne). 7:150. https://doi.org/10. 3389/fendo.2016.00150

20. Liang Z, Zhu H, Wang X et al (2020) Adjuvants for coronavirus vaccines. Front Immunol 11:589833. https://doi.org/10.3389/ fimmu. 2020.589833

21. Lui DTW, Lee KK, Lee CH, Lee ACH, Hung IFN, Tan KCB (2021) Development of Graves' disease after SARS-CoV-2 mRNA vaccination: a case report and literature review. Front Public Health 9:778964. https://doi.org/10.3389/fpubh.2021.778964

22. Bragazzi NL, Hejly A, Watad A, Adawi M, Amital H, Shoenfeld Y (2020) ASIA syndrome and endocrine autoimmune disorders. Best Pract Res Clin Endocrinol Metab 34(1):101412. https://doi. org/10.1016/j.beem.2020.101412

23. Colafrancesco S, Perricone C, Tomljenovic L, Shoenfeld Y (2013) Human papilloma virus vaccine and primary ovarian failure: another facet of the autoimmune/ inflammatory syndrome induced by adjuvants. Am J Reprod Immunol 70:309-316. https://doi.org/ 10.1111/aji.12151

24. Alvarez-Soria MJ, Hernandez-Gonzalez A, Carrasco-Garcia de Leon S, del Real-Francia MA, Gallardo-Alcaniz MJ, LopezGomez JL (2011) Demyelinating disease and vaccination of the human papillomavirus. Rev Neurol 52:472-476

25. Olivieri B, Betterle C, Zanoni G (2021) Vaccinations and autoimmune diseases. Vaccines (Basel). 9(8):815. https://doi.org/10. 3390/vaccines 9080815

26. COVID-19_Actualizacion9_EstrategiaVacunacion.pdf (mscbs. gob.es)

27. World Health Organization. Immunization Safety Surveillance: guidelines for immunization programme managers on surveillance of adverse events following immunization, 3rd ed.; WHO: Geneva, Switzerland, 2016; pp. 1-169

Publisher's Note Springer Nature remains neutral with regard to jurisdictional claims in published maps and institutional affiliations. 